\title{
LES ÉCRIVAINS CONTRE L'ETHNOLOGIE ? ETHNOGRAPHIE, ETHNOLOGIE ET LITTÉRATURE D’AFRIQUE ET DES ANTILLES, 1921-1948
}

Donc, camarade, te seront ennemis - de manière haute, lucide et conséquente - non seulement gouverneurs sadiques et préfets tortionnaires, non seulement colons flagellants et banquiers goulus [...], mais pareillement et au même titre, [. . .] ethnographes métaphysiciens et dogonneux, théologiens farfelus et belges, intellectuels jaspineux, sortis tout puants de la cuisse de Nietzsche ou chutés calenders-fils-deRoi d'on ne sait quelle Pléiade [. . .] - tous suppôts du capitalisme, tous tenants déclarés ou honteux du colonialisme pillard, tous responsables, tous haïssables, tous négriers, tous redevables désormais de l'agressivité révolutionnaire. (Césaire 31)

C es quelques lignes ouvrent le quatrième chapitre du Discours sur le colonialisme d'Aimé Césaire. Cette partie du livre s'attache à montrer que le colonialisme ne se réduit pas aux formes les plus manifestes de la violence politique, mais qu'il existe un colonialisme subreptice, plus insidieux, inscrit dans les discours savants de l'époque. "Ethnographes métaphysiciens et dogonneux ": allusion à l'ethnologue Marcel Griaule et à son école, qui depuis les années 1930, se sont fait une spécialité de la "métaphysique » ou de la «sophie » des populations africaines, en particulier des Dogons, illustrée par le très populaire Dieu d'eau paru en 1948. "Théologiens farfelus et belges " : allusion au missionnaire franciscain Placide Tempels et à sa Philosophie bantoue parue en 1945, qui tâchait de dégager, à la source de l'univers mental bantou, une ontologie de la force vitale ${ }^{1}$. "Intellectuels jaspineux, sortis tout puants de la cuisse de Nietzsche ou chutés calenders-fils-de-Roi d'on ne sait quelle Pléiade » : allusion vraisemblable à Roger Caillois, ancien étudiant de Marcel Mauss à l'Institut d'ethnologie de Paris, cofondateur (avec Georges

1. Sur Tempels et sa lecture par les auteurs de la négritude, voir Miller 11-14. Pour une relecture et une réhabilitation récente de Tempels, voir Diagne, L'Encre des savants 21-52.

The Romanic Review Volume 104 Numbers 3-4 (C) The Trustees of Columbia University 
Bataille et Michel Leiris) du Collège de Sociologie, grand lecteur de Nietzsche, et ici comparé aux voyageurs « fils-de-roi » des Pléiades d'Arthur de Gobineau, lui-même auteur de l'Essai sur l'inégalité des races humaines.

Voilà donc trois figures qui, en apparence, désignent l'ethnologie comme l'ennemi de l'écrivain noir révolutionnaire et l'une des cibles du combat anticolonial. Et c'est aujourd'hui un postulat implicite de nombreuses études postcoloniales de la littérature francophone : l'ethnologie est une discipline irrémédiablement discréditée par son inscription dans l'histoire impériale, comme l'attestent pêle-mêle les pratiques de mesure des corps et des crânes qui se poursuivent encore au début du 20e siècle, la participation du Musée d'ethnographie du Trocadéro à l'Exposition coloniale internationale de 1931, les vitrines du Musée de l'homme où voisinent artefacts et ossements jusqu'aux années 1950, ou l'abstraction réductrice de l'anthropologie structurale, toutes pratiques et discours porteurs d'une même violence " coloniale » et d'une même logique de réification de l' « autre ». Il est tacitement entendu que les premiers auteurs francophones issus des colonies n'ont pu que se révolter contre une telle entreprise et que leurs œuvres constituent une réponse et un contre-discours. They wrote back.

Pourtant la précision des références de Césaire devrait inciter à plus de prudence. Elle semble indiquer qu'il est très au fait des développements de la discipline ethnologique, et qu'il en a une connaissance suffisamment fine pour ne pas la rejeter en bloc comme un discours homogène. C'est d'abord une question de date. Foucault et ses analyses de l'intrication entre savoirs et pouvoir ne sont pas encore passés par là. Césaire est plus classiquement marxiste ; son propos n'est pas d'identifier une violence symbolique inhérente à toute entreprise de connaissance, il est de repérer des complicités et de révéler des idéologies. Mais c'est aussi que, comme beaucoup de ces contemporains, Césaire voit d'abord le discours ethnologique comme une chance et une promesse. Pour la première fois, un relativisme culturel radical s'y développe et s'y épanouit, non comme idéologie critique ou comme hypothèse théorique à la lisière de la provocation (comme au temps des avant-gardes du début du siècle), mais avec la caution de l'institution universitaire et l'autorité sereine de la science - cette science qui, comme l'écrit Roland Barthes exactement la même année, "va vite et droit en son chemin ", laissant derrière elle des « représentations collectives [qui] ne suivent pas ... maintenues stagnantes dans l'erreur par le pouvoir, la grande presse et les valeurs d'ordre » :

[. . .] les efforts des ethnologues pour démystifier le fait nègre, les précautions rigoureuses qu'ils observent déjà depuis fort longtemps lorsqu'ils sont obligés de manier ces notions ambiguës de "Primitifs " ou d' "Archaïques ", la probité intellectuelle d'hommes comme Mauss, Lévi-Strauss ou Leroi-Gourhan aux 
prises avec de vieux termes raciaux camouflés, on comprendra mieux l'une de nos servitudes majeures : le divorce accablant de la connaissance et de la mythologie. (Barthes 67)

Dans la même veine, la suite du chapitre du Discours sur le colonialisme est en réalité une défense sinon des pratiques ethnographiques (Césaire tolère les musées d'ethnographie, mais considère qu' " il eût mieux valu, à tout prendre, n'avoir pas eu besoin de les ouvrir »), du moins de la discipline et de son relativisme fondamental, contre les attaques de Caillois, dans "Illusions à rebours ". Dans ce long essai paru dans la conservatrice Nouvelle Nouvelle Revue française, Caillois attaquait (avec deux ans de retard) les thèses de Claude Lévi-Strauss dans Race et Histoire. Il s'agissait en gros, pour Caillois, d'accepter une certaine dose de relativisme sur des bases morales, faites de tolérance et d'ouverture d'esprit, mais de présenter la position relativiste de Lévi-Strauss comme contradictoire et théoriquement intenable, avant d'accorder à la civilisation occidentale un privilège (Caillois ne dit pas "supériorité », mais c'est cela qu'il pense) qui tient précisément au fait qu'elle est la seule à avoir des ethnographes et à faire montre dans ses discours et ses institutions d'un intérêt pour les sociétés " autres ». Lévi-Strauss avait répondu dans Les Temps modernes avec un article rigoureux, qui interrogeait la possibilité du relativisme culturel comme position théorique et répondait systématiquement aux arguments de Caillois, mais d'une telle virulence qu'il renoncerait à l'inclure trois ans plus tard dans Anthropologie structurale $^{2}$. C'est dans ce même contexte que Césaire écrit son Discours sur le colonialisme et que, sur un mode non moins virulent, il s'insurge contre les études philosophiques et savantes qui prétendent isoler les causes objectives (sociologiques, psychiques, religieuses ....) du prétendu " retard » des sociétés colonisées et expliquer le colonialisme et la marginalisation du "non-Blanc » autrement que par l'expansion du capitalisme marchand et l'exploitation économique. Contre Caillois, Césaire mobilise donc les travaux de Leiris et de Lévi-Strauss et il cite même l'édition, qu'on ne peut pourtant pas dire grand-public, des Carnets posthumes de Lucien Lévy-Bruhl, auteur voué aux gémonies pour avoir caractérisé la "mentalité primitive » comme

2. Voir Lévi-Strauss. On peut rétrospectivement regretter cette absence du recueil Anthropologie structurale, car le caractère prétendument intenable du relativisme anthropologique est aujourd'hui un postulat implicite des formes les plus vulgaires de la théorie postcoloniale alors que cette question théorique difficile, loin d'être ignorée des ethnologues, a une longue histoire et son traitement par Lévi-Strauss en est un des développements les plus remarquables. Sur Race et Histoire, ouvrage plus complexe que sa réputation de catéchisme antiraciste ne le laisse penser, voir Stoczkowski. 
" prélogique », mais dont les derniers écrits montrent qu'il avait renoncé à cette notion peu avant sa mort $^{3}$.

Publié trente ans après la fondation de l'Institut d'ethnologie de Paris, ce passage du Discours sur le colonialisme rappelle qu'en réalité pour les premiers écrivains francophones issus des colonies françaises, le rapport à l'anthropologie a souvent été complexe - ni adhésion enthousiaste, ni rejet massif. Les premières œuvres de ce qu'on appelait jusqu'aux années 1950 la « littérature indigène d'expression française » sont en effet contemporaines du développement de l'ethnologie comme discipline universitaire (qu'on peut dater, en France, de la fin des années 1920 et des années 1930). Or loin de rejeter ce nouveau discours comme un savoir colonial qui réifie ses objets, ces auteurs et écrivains ont fait montre d'un grand intérêt pour cette discipline ; ils l'ont souvent étudiée, et ont parfois activement contribué à son développement. Dans une importante étude consacrée aux premières «fictions ethnographiques africaines »(René Maran, Paul Hazoumé, Chinua Achebe), Elini Coundouriotis montrait déjà que ces ouvrages ne devaient pas être lus comme des œuvres de "résistance " aux discours savants, selon le prisme d'une " opposition entre l'Europe et ses autres »; elle proposait d'y voir à l'œuvre plutôt un "paradigme de dissidence », les écrivains noirs d'Afrique et des Antilles utilisant la littérature pour réintroduire, dans les représentations des sociétés colonisées, une historicité qui manquait au discours africaniste dominant (Coundouriotis 20). Cette hypothèse selon laquelle les écrivains indigènes ont utilisé la littérature pour "réclamer l'histoire » n'est pas inexacte, mais elle reste très générale et elle est insuffisamment attentive à l'inscription des écrivains dans le tissu discursif colonial ainsi qu'à la complexité des constructions auctoriales en situation de domination. Il est tentant de considérer les fictions littéraires comme une subversion du discours savant, mais en l'occurrence, ce serait projeter une conception (post)moderne de la littérature sur une altérité historique qui obéissait à des découpages discursifs et à des logiques de subjectivation qui n'ont que peu à voir avec l'opposition entre science et littérature telle qu'elle a été forgée par la critique post-structuraliste des années 1960 ou 1970.

Faute de pouvoir détailler, à propos de chaque cas particulier, l'articulation entre ethnologie et littérature des colonies dans les années 1930, je propose de décrire quelques dynamiques générales, à la fois sociologiques et théoriques, qui aident à comprendre certaines trajectoires individuelles. Je considérerai ici quelques auteurs antillais mais surtout la première génération d'auteurs africains francophones, entre la publication de Batouala (1921) et celle de l'Anthologie de la poésie nègre et malgache (1948), en insistant sur la singularité

3. Pour une relecture théorique de l'œuvre de Lévy-Bruhl, voir Keck. 
de cette configuration et en gardant en tête qu'il ne s'agit là que d'une partie d'un tableau plus complet : un travail semblable est nécessaire à propos des premiers auteurs francophones du Maghreb et de ceux de l'Indochine.

$$
* * *
$$

\section{Africains et Antillais à l'Institut d'ethnologie}

L'Institut d'ethnologie de Paris est fondé en 1925. C'est une petite structure interne à la Sorbonne, qui à partir de 1927 délivre un certificat d'ethnologie valable pour la licence ès lettres, et à partir de 1928 un autre valable pour la licence ès sciences (il faut, à l'époque, quatre certificats pour obtenir sa licence). L'ethnologie est une discipline dynamique et attrayante pour beaucoup d'étudiants parisiens de la "génération de 1905 ». Elle incarne une forme de nouveauté intellectuelle et elle est portée par le financement généreux des gouvernements des colonies, par une politique de popularisation de ces résultats (en particulier autour du musée), par sa proximité avec le monde de l'art, et par les promesses d'évasion qu'elle porte 4 . Les cours donnés à l'Institut sont populaires - on compte jusqu'à 170 élèves et auditeurs inscrits par an dans les années 1930 - mais seule une petite minorité passe effectivement l'un des deux certificats. Or parmi les premiers certifiés (on en compte entre 10 et 15 par an pour chaque certificat), on trouve non seulement les membres de la " première génération » de l'ethnographie française (Alfred Métraux, Marcel Griaule, Jacques Soustelle, Denise Paulme, André Schaeffner, Germaine Tillion, Michel Leiris) mais aussi les noms de plusieurs auteurs en devenir, issus de l'Empire français : le lettré malgache Albert Rakoto Ratsimamanga est certifié en juin 1934 ; le romancier dahoméen Paul Hazoumé l'est en novembre 1938 ; Léopold Sédar Senghor l'est en juin $1939^{5}$. L'écrivain communiste haïtien Jacques Roumain est certifié en juin 1938 et il fondera trois ans plus tard l'Institut d'ethnologie de Port-au-Prince. Certes Haïti n'est pas une colonie française, mais la présence de Roumain est indicative de l'intérêt que la nouvelle discipline suscite chez des intellectuels antillais, comme le montre également le parcours de Suzanne Comhaire-Sylvain qui a suivi la formation de Mauss non

4. Voir Laurière ; Debaene, L'Adieu ; Conklin, In the Museum.

5. Source : Archives de l'Institut d'ethnologie à la Bibliothèque centrale du Muséum d'histoire naturelle de Paris. AMH/IE/2AM 2 A 1. Il ne m'a malheureusement pas été possible de retrouver dans les archives les listes des auditeurs, et donc de déterminer l'origine des étudiants inscrits à l'Institut d'ethnologie entre 1925 et 1939, pourtant dix ou douze fois plus nombreux que les certifiés dont seuls les noms apparaissent dans les procès-verbaux des séances du conseil de l'Institut. 
à l'Institut d'ethnologie mais à l'École pratique des hautes études, et dont les deux thèses sur le créole et les contes haïtiens sont publiés en 1936 et $1937^{6}$.

L'itinéraire du poète guyanais Léon-Gontran Damas en offre une illustration plus parlante encore : il suit les cours de Marcel Mauss à l'Institut d'ethnologie, et en 1934, alors qu'il n'a pas encore terminé sa formation, il est chargé par Mauss et Paul Rivet (le directeur du Musée d'ethnographie du Trocadéro) d'une mission d'étude des "nègres bosch ", dans le cadre d'un projet d'exposition «Africa in America », initié par l'anthropologue allemand Paul Kirchhoff. La contribution de Damas aurait dû enrichir la troisième section de l'exposition que le projet, rédigé en anglais par Kirchhoff, décrivait en ces termes : " [devoted to] a third group : rebellious and run-away negroes either returning to a more or less African type of life, or founding free "republics" [. . . . It will range from the Bush-negroes in Surinam, molding their life after the African model, only on a lower scale, to the revolution in San Domingo, resulting in the setting-up of a modern state, and from the earliest known risings and free republics in Brazil in the first century after the beginning of slave importation, to the modern struggle against the occupation of Haït ${ }^{7}$. Damas séjourne effectivement auprès de sept tribus marrons de Guyane, et en revient avec un recueil de contes, publié tardivement et intitulé Veillées noires, mais son livre Retour de Guyane, paru en 1938, mentionne à peine sa mission ethnographique, à l'origine pourtant de son voyage sur ses terres natales. Pour des raisons obscures, qui tiennent sans doute à l'histoire chaotique du Musée d'ethnographie à partir de 1935 (il sera démoli pour laisser place au Musée de l'homme) ainsi qu'au départ de Kirchhoff (qui tente de quitter l'Europe et trouve alors un refuge éphémère à Columbia University), le projet d'exposition est abandonné. Quant à Damas, il ne publiera aucun texte issu de son ethnographie des nègres marrons, ni ne s'expliquera sur ce point, se contentant de laisser entendre qu'il a été saisi par l'urgence du témoignage sur la Guyane coloniale : "Vivre au jour le jour, des mois durant, la vie matérielle et sociale de ces nègres restés purs ; recueillir toute documentation ; tel était le but de ma mission. Volontiers m’y fussé-je cantonné si, parallèlement, je

\footnotetext{
6. Les travaux de Roumain et Comhaire-Sylvain s'inscrivent dans une histoire ancienne puisque la réflexion anthropologique en Haïti remonte au dix-huitième siècle, mais celle-ci connaît un "tournant ethnologique ", selon l'expression de Carlo Avierl Célius, à la suite de la publication de Ainsi parla l'oncle de Jean Price-Mars en 1928 (Célius). Voir aussi Magloire et Yelvington.

7. AMH/IE/2AM 1 K54c. Dossier Paul Kirchhoff, projet d'exposition "Survivances africaines dans le Nouveau Monde ». Dans sa préface à la réédition de Retour de Guyane, Sandrine Poujols signale que Mauss et Rivet auraient sollicité également Jean Price-Mars, l'anthropologue américain Melvin Herskovits et le Cubain Fernando Ortiz (Poujols 13).
} 
n'avais eu à me pencher sur le problème que pose la Guyane française [. . .] » (Damas 27) ${ }^{8}$.

Quoi qu'il en soit, cette implication de sujets colonisés au capital scolaire élevé (la licence est, à l'époque, un examen réservé à une étroite élite) dans les institutions de la nouvelle discipline ethnologique intrigue. Elle montre que l'Institut d'ethnologie est un endroit parmi d'autres où se croisent intellectuels africains et antillais et où la race se met en jeu et s'invente pour la diaspora noire parisienne (voir Edwards). En 1931, la Revue du monde noir invitait ses lecteurs à assister aux cours de Mauss, "le savant sociologue disciple de Durkheim », au Collège de France sur la notion de " primitif » ( Les sciences sociologiques»191), et quelques mois plus tard, Paulette Nardal indiquait que ce serait " avec l'aide des savants de race blanche et de tous les amis des Noirs " qu'il serait possible de " redonner à nos congénères la fierté d'appartenir à une race dont la civilisation est peut-être la plus ancienne du monde » (Nardal 349). En considérant le rapport à l'ethnologie des auteurs africains qui commencent à écrire dans les années 1930, il s'agit donc d'éclairer l'un des « amonts » de cette histoire.

\section{L'indigène "dans la vérité de ses gestes et l'intimité de sa pensée »}

"The only avenue of publication consistently available for African writers was literature ", écrit Elini Coundouriotis. "One of the shaping impulses behind their fiction was to counter the authority of European social 'scientists' who studied Africa and Europe's relation to Africa. » (Coundouriotis 14) Une telle description suppose un espace de publication ouvert et, pour l'indigène lettré, une liberté dans le choix du genre qui sont loin de la réalité historique de la domination coloniale. Dans les faits, c'est presque l'inverse qui est vrai : la littérature (sous la forme de fictions, d'essais ou de recueils de poèmes) n'est pas vraiment, avant la fin des années 1930, une forme d'expression possible pour l'indigène francophone. Dans les écrits des administrateurs, professeurs et autres représentants de l'autorité coloniale, elle n'est au mieux envisagée que comme une possibilité lointaine. La première voie - et pour beaucoup d'auteurs, la seule qui soit ouverte - fut au contraire l'ethnographie, et bien souvent, ce sont les contributions ethnographiques qui ont autorisé la "prise de parole » littéraire.

On peut considérer que la possibilité d'une littérature africaine d'expression française est l'aboutissement de deux logiques convergentes - et que toutes deux accordaient une place essentielle à l' " ethnographie ", qu'il faut

8. Dans ses entretiens avec Daniel Racine, Damas dit que les « résultats » de sa mission ont été déposés au Musée de l'homme (Musée d'ethnographie à l'époque), mais il m'a été impossible de les retrouver dans les archives (Racine 197). 
moins entendre, en l'occurrence, comme une discipline universitaire, avec ses méthodes et son histoire, que comme l'étude des mœurs et coutumes de sociétés posées comme "autres". La première de ces dynamiques relève de la " littérature coloniale ", ce vaste complexe discursif et symbolique, constitué d'une colossale production de fictions et d'anthologies, mais aussi de théories et d'institutions (le Grand prix de littérature coloniale, etc.). Le cœur en est le roman " réaliste ", écrit à des fins de propagande (le mot n'est pas insultant avant la fin des années 1930), " pour faire aimer nos colonies » (c'est le titre d'une collection des éditions Peyronnet), par des hommes qui sont presque toujours eux-mêmes membres de l'administration coloniale et signent souvent d'un nom de plume. Citons pour mémoire Robert Randau (né Robert Arnaud), les frères Tharaud, les cousins Marius et Ary Leblond (Georges Athénas et Alexandre Merlot), Louis Bertrand, René Maran, André Demaison, Georges Hardy, Louis Faivre (Robert Delavignette), Pierre Mille, Eugène Pujarniscle. ... C'est un continent à la fois oublié et immense (plusieurs de ces titres ont été vendus à plus de cent mille exemplaires; le catalogue de la Bibliothèque nationale de France attribue 349 notices à Jean Tharaud et 361 notices à son frère Jérôme . . .) et comme l'ont très bien montré plusieurs critiques dès les années 1980, c'est ce vaste ensemble qui constitue l'environnement immédiat, à la fois idéologique et esthétique, de la première littérature africaine en français 9 .

Cette littérature coloniale s'organise historiquement comme une sorte de " micro-champ » au sens de la sociologie de Bourdieu. C'est un espace constitué et déterminé par la rivalité pour le monopole du discours sur les colonies. Il s'agit d'un domaine traversé de tensions dont l'enjeu organisateur est la connaissance vraie, exacte, approfondie de l'outre-mer - comme l'attestent par exemple les incessantes attaques contre les journalistes, les romanciers exotiques (Loti au premier chef) ou les « écrivains touristes » qui " ne font que passer"; comme l'attestent plus encore les virulentes polémiques qui font suite à la publication de Batouala, éloquemment soustitré « véritable roman nègre ", à quoi répondront (entre autres) Gaston Joseph avec Koffi, roman d'un vrai noir ou Maurice Delafosse qui, dans Broussard ou les états d'âme d'un colonial, s'insurge contre un ouvrage qui « n'est ni véritablement un roman, ni véritablement nègre » (Delafosse, Broussard $137)^{10}$. Cette structuration de l'espace du discours a pour conséquence idéologique (et même "épistémologique » en un sens) une spatialisation de l'altérité : le voyageur qui ne fait que passer est condamné à une connaissance

9. Voir en particulier Steins ; Midiohouan, L'Idéologie ; Miller.

10. Pour une analyse fine du sous-titre de Batouala et de ses implications, voir Edwards 81-93. 
superficielle de l'Afrique et des Africains ; le romancier exotique, ébloui par la nouveauté des paysages, ne dépasse pas la description toute extérieure ; seul le « vrai colonial » (Robert Randau, né en Algérie et «véritable Africain d'adoption ", ou Maurice Delafosse pour lequel la France n'est qu'une "station de repos » entre deux missions) pénètre l'âme indigène et peut prétendre à la connaissance intime des cœurs et des esprits (Lebel 229-30 ; voir aussi Pujarniscle 14-17). Cette distribution en termes de profondeur va de pair avec une histoire, qui est celle de l'expansion coloniale elle-même : de même qu'il a fallu d'abord conquérir les côtes du continent, puis l'intérieur des terres, avant de finalement gagner les cœurs et l'âme indigènes, la littérature coloniale a connu un développement à plusieurs étapes : d'abord, " une littérature de voyage et de conquête ", puis une "littérature technique " (notices de géographie, traités d'histoire, études d'économie ...), avant, enfin, "la littérature d'imagination ", plus soucieuse de psychologie et dont le roman colonial est l'aboutissement ${ }^{11}$.

Les indigènes sont à peu près exclus de cet espace jusqu'aux années 1930, mais leur participation était en quelque sorte programmée, nolens volens, par cette construction. Si Roland Lebel dans sa thèse n'envisage pas explicitement la possibilité d'une littérature indigène d'expression française, elle découle naturellement de son idéal d'une littérature coloniale «fortement enracinée dans le sol qui la nourrit ", qui montre "l'élément indigène dans la vérité de ses gestes et l'intimité de sa pensée » (234). Eugène Pujarniscle, lui, consacre les dernières pages de son Philoxène ou de la littérature coloniale à ce qu'il considère explicitement comme un point d'arrivée, à savoir l'auteur indigène qui décrit sa propre société et que "rien ne sépare de l'objet de son étude » (187). Au terme d'un long développement (d'une condescendance pénible et d'un racisme patent), il indique que même s'il serait " excessif » de " conclure que l'avenir de la littérature coloniale est entre les mains des indigènes ", celle-ci peut néanmoins " être complètement renouvelée par l'apport indigène » $(185-6,201)$. Dans cette perspective, on voit que les premières fictions africaines en français, loin d'être un lieu de contestation de l'autorité, constituent plutôt un aboutissement logique. Elles sont bien l'expression du " point de vue indigène ", mais un point de vue qui ne s'oppose à aucune perspective extérieure ; il s'agit au contraire d'accomplir ce projet de savoir qui doit permettre d'asseoir la domination en l'appuyant sur une connaissance intime des "réalités morales » locales. Ce n'est certes pas à des fins de subversion que le bandeau publicitaire de L'Empire du Mogho-Naba, ouvrage de Dim Delobsom préfacé par Robert Randau, proclamait : «Un Empire noir

11. C'est ainsi qu'est organisée la thèse de Roland Lebel, L'Afrique occidentale dans la littérature française (depuis 1870), parue en 1925. 
vu par un Noir ${ }^{12}$. Les avant-propos des administrateurs et autres patrons aux premiers textes africains sont d'ailleurs particulièrement explicites sur ce point : Georges Hardy célèbre dans Doguicimi « un drame qui nous porte au cœur même de la société locale et nous familiarise progressivement avec ses démarches de pensée », mais il a commencé par préciser que Paul Hazoumé est un " honnête homme ", qui ne conçoit "d'autre patrie possible que la nôtre ": "vous l'étonneriez fort si vous lui prêtiez imprudemment la moindre visée autonomiste. » (Hardy 10-11) Le roman Karim d'Ousmane Socé, paru initialement en 1935, est sans doute le meilleur exemple de cette continuité entre littérature coloniale à préoccupation ethnologique et littérature africaine (voir Midiohouan, "Littérature africaine»).

\section{La littérature ethnographique de "nos meilleurs éléments "}

La seconde dynamique conduisant à la naissance d'une littérature indigène en français passe plus directement par l'ethnographie. À partir des années 1920 et plus encore des années 1930, l'administration entend fonder la colonisation sur la « connaissance directe de l'indigène ", la " méthode expérimentale " (par opposition à la politique "théorique » déterminée dans les ministères), et le respect des coutumes locales. Dans les faits, ce nouvel " humanisme colonial » est très conservateur et, sous couvert de " rapprochement avec les administrés indigènes ", conduit à renforcer l'étanchéité entre élites colonisées et administration coloniale (voir Conklin, A Mission 174-245 ; de l'Estoile, "Science de l'homme "; Wilder 118-45). Mais cette réorientation politique stimule explicitement la production d'un discours ethnographique indigène ${ }^{13}$. Celui-ci se développe dans différents cadres : à l'école (où le mémoire sur les mœurs ou les coutumes de la société d'origine fait partie de la formation, à l'exemple des « devoirs de vacances » de l'École normale William-Ponty) (voir Warner) ; dans le cadre de la justice coloniale, lorsque les tribunaux locaux doivent juger une affaire en s'appuyant sur les « coutumiers » existants (voir Jézéquel, «Collecting ») ; ou plus généralement lorsque l'autorité coloniale souhaite faire valoir l'efficacité de son œuvre de civilisation. Ces contributions sont en effet toujours encadrées par le discours d'un parrain - professeur, administrateur ou missionnaire - qui les

12. On retrouve ici les conclusions d'Edward Said dans « Representing the Colonized ", puisque le point de vue de l'indigène n'est pas d'abord un fait ethnographique ou une simple construction herméneutique, mais une construction du projet anthropologique lui-même qui le constitue à la fois comme un terme (à atteindre) et comme un objet (à réduire). (Voir Said 219-20)

13. Pour une chronologie plus fine et la situation de ce renouveau des études indigènes après un premier moment dans les années 1910, voir Jézéquel, « Voices ». 
introduit, les justifie et en souligne les mérites ${ }^{14}$. C'est que, comme l'écrit Anne Piriou, " les prouesses d'instituteurs érudits, preuves vivantes de la réussite de l'enseignement, témoign[ent] hautement des vertus de l'école civilisatrice. [...] Elles sont avant tout des faire-valoir d'une politique de rayonnement de la langue française. » (Piriou 65-66). Robert Randau décrit Dim Delobsom comme un de ces " noirs évolués » qui ont "adopt[é], au moins en partie, nos façons de penser » et qui " qualifient volontiers de sauvages les gens qui obéissent intégralement à la coutume ethnique »(Randau i) ; Gabriel Monod-Herzen voit dans le style de Fily Dabo Sissoko un " témoignage et de ce que valent certaines écoles françaises de l'Afrique, et des possibilités intellectuelles des Noirs » (Monod-Herzen 246). Ces écrits indigènes servent donc d'arguments dans les " luttes de concurrence internes à l'entreprise de colonisation » (de l'Estoile, "Science de l'homme » 313) et dans les échanges des agents coloniaux avec l'administration métropolitaine, avec le pouvoir politique et parlementaire, ou avec les centres universitaires parisiens auprès desquels ils essaient de faire valoir une expertise qui leur est propre (voir Piriou ; Sibeud ; Debaene, "Entre informateur »).

Pour beaucoup d'Africains de "l'élite », fils de chef ou membres de l'aristocratie indigène, qui naissent au moment de la conquête et dont la famille ou le clan sont en rapport étroit avec l'administration française, l'ethnographie va ainsi constituer la première et parfois l'unique forme possible d' « expression » - terme problématique en l'occurrence puisqu'il s'agit d'un discours sollicité. Les tout premiers textes francophones africains ne sont ni des romans, ni des pièces de théâtre, encore moins des poèmes, mais des études linguistiques et ethnographiques rédigées à la demande de l'administration par des interprètes ou des instituteurs formés par les missionnaires ou les institutions coloniales. Le lettré "voltaïque » Dim Delobsom, l'instituteur Mamby Sidibé ou l'interprète "soudanais " Moussa Travélé, nés dans les années 1880 ou 1890, accèdent à la visibilité non par des fictions, mais par des articles savants sur les rites ou les coutumes qui paraissent dans les périodiques coloniaux, avec bien souvent l'explicite volonté de contribuer " à la connaissance de plus en plus parfaite de la psychologie des races ouest-africaines pour pouvoir mieux seconder l'action française en ce pays » (Sidibé 72-73) ${ }^{15}$. La publication de ces écrits ethnographiques constitue la première étape vers l'émergence d'un espace public colonial qui fut lui-même

14. La signature de l'indigène est ainsi systématiquement redoublée de celle de son " patron ». Pour une analyse de ces parrainages et de ce phénomène de double signature, voir Debaene « Entre informateur».

15. Sur les motivations complexes et variables qui peuvent présider à ces contributions indigènes, voir Jézéquel, « Voices ». 
la condition de la première littérature africaine francophone ${ }^{16}$. Paul Hazoumé, né en 1890, sera le premier auteur à passer à la littérature après avoir « fait ses preuves " avec des études ethnographiques parues d'abord dans la presse coloniale puis dans la série Travaux et Mémoires de l'Institut d'ethnologie (Le Pacte de sang au Dahomey), une trajectoire qu'on retrouve également chez Fily Dabo Sissoko, né en 1900, ou Ibrahima Mamadou Ouane, né en 1908, eux aussi anciens élèves de l'École William-Ponty.

Loin d'être un discours savant auquel les auteurs africains vont s'opposer, l'ethnographie a donc plutôt constitué un préalable. Comme l'a remarqué János Riesz à propos de Doguicimi, elle a pu opérer comme un " prérequis ", une sorte de droit d'entrée à acquitter avant "l'expression française » sous forme de fictions. "Dans des disciplines savantes comme l'ethnographie et l'historiographie, il était plus facile d'exercer un contrôle strict sur les auteurs indigènes », explique-t-il, et limiter leur prise de parole à cet espace contraint était sans doute une façon de s'assurer de leur loyauté avant de leur accorder ou non un exercice plus libre du discours (Riesz 17, 30). Georges Hardy décrit ainsi en 1931 les « travaux historiques » rédigés par des indigènes (à l'exemple du Pacte de sang au Dabomey, alors paru seulement en feuilleton) comme « une excellente préparation »: " un jour ou l'autre, tous ces jeunes gens nous donneront certainement une littérature d'esprit indigène, mais en langue française. " (Congrès international 203) Mais peut-être ce rôle de l'ethnographie comme " préparation » à la littérature est-il plus profond encore. Plus qu'un simple marchepied, ou qu'un passage obligé, on peut se demander si l'écrit ethnographique n'est pas l'occasion d'une «fabrique de l'auteur » car en même temps qu'il garantit, pour l'autorité coloniale, une bonne distance à l'égard de la culture d'origine, il produit aussi cette " pluralité d'ego » nécessaire à la constitution de la fonction-auteur (Foucault, "Qu'est-ce qu'un auteur » 831). Pour l'administrateur, la compétence ethnographique est en effet le gage d'un sujet colonial moderne, qui a construit un rapport " équilibré » à ses origines indigènes : il les envisage avec sympathie - " il entend ne point se détacher inutilement du sol de ses ancêtres, du passé de sa famille, des habitudes de son entourage »-, mais il en connaît aussi les insuffisances et il sait qu'une modernisation menée sous tutelle française est inéluctable et doit conduire à " une amélioration d'existence sans déracinement » (Hardy 10). Autrement dit, l'injonction ethnographique relève très précisément d'une logique d'assujettissement telle que Foucault la décrit dans Surveiller et Punir : elle produit des subjectivités en exerçant son pouvoir et exerce son pouvoir en produisant des subjectivités. Il n'y a pas un sujet préalablement donné sur lequel s'exerce la domination (et qui, ensuite, " répliquerait » [write back]),

16. Sur cette notion d'espace public colonial, employée entre autres par Lüsebrink, et sur ses implications, voir Jézéquel, « Les enseignants ». 
mais la constitution par le dispositif de savoir-pouvoir d'un sujet qui sera à la fois le lieu où s'appliquera de la discipline et le lieu d'où émanera la résistance au pouvoir. En exigeant que l'auteur soit à la fois fidèle à ses origines et distant de ses origines, c'est-à-dire en réclamant l'intériorisation d'une injonction d'authenticité, l'institution coloniale produit le sujet lettré comme dédoublement ${ }^{17}$. Ce n'est pas, en tant que telle, une condition de l'auctorialité, mais c'est sans doute une condition de l'auctorialité littéraire moderne : il faut un sujet, à la fois unique et pluriel, qui puisse se constituer comme origine de l'œuvre. Dans cette perspective, il faudrait alors dire que, très paradoxalement, loin d'être le contre-modèle de la littérature comme l'inventaire s'oppose au récit ou comme la structure s'oppose à l'histoire (voir Coundouriotis 13-16), l'ethnographie - ou en tout cas l'injonction ethnographique - en a été l'une des conditions.

Il faut enfin noter que ce n'est pas n'importe quelle littérature qui est ainsi rendue possible mais, dans un premier temps en tout cas, une littérature représentative, "réaliste ", et qui privilégie certains thèmes et certains arcs narratifs, en particulier la " petite patrie » ou l'opposition entre tradition et modernité ${ }^{18}$. La négritude constituera une rupture d'abord pour cette raison : le privilège accordé à la poésie lyrique sort d'une littérature vouée à la connaissance du Noir par sa représentation. Elle ne rompra pas pour autant avec l'ethnographie, bien au contraire, mais la retrouvera par un autre biais, à savoir le recueil de folklore et les adaptations et traductions sur le modèle des Poèmes nègres sur des airs africains publiés par Damas en 1947, des Contes d'Amadou Koumba de Birago Diop (1947) ou des Contes et légendes d'Afrique noire qu'Ousmane Socé publie à la suite de la réédition de Karim en 1948.

\section{«Près des remparts rebâtis de ma mémoire ${ }^{19}$. "}

L'histoire des « élites lettrées » indigènes est une histoire chronologiquement extrêmement resserrée, qui impose une lecture en termes générationnels. Une vingtaine d'années séparent les auteurs nés au moment de la conquête, comme

17. Voir Debaene, «Entre informateur ». Ce point est particulièrement mis en évidence par la passionnante étude des Cahiers Ponty par Toby Warner, qui montre de façon éloquente comment l'exercice du mémoire ethnographique est l'occasion d'une subjectivation par négociation et recomposition de l'injonction scolaire. Pour une analyse très similaire des écrits féminins de la même période, voir Barthélémy.

18. Pour une étude détaillée de ces thèmes dans les premiers écrits féminins, voir Barthélémy. Sur les modèles littéraires qui imprègnent les Cahiers Ponty, et particulièrement le Bildungsroman, voir Warner. Voir aussi Midiohouan, "Littérature africaine ". 19. "J'ai choisi ma demeure près des remparts rebâtis de ma mémoire, à la hauteur des remparts. "C'est ainsi que, dans Chants d'ombre, Senghor décrit son installation 
Travélé, Hazoumé ou Sidibé, et la génération de Senghor et Birago Diop, nés en 1906, qui eux-mêmes font figure d'aînés auprès d'Ousmane Socé, né en 1911, mais aussi de Damas et Césaire (nés respectivement en 1912 et 1913), lorsqu'ils se rencontrent à Paris au début des années 1930. Si l'on suit les écrits publiés par les étudiants de l'École normale fédérale de l'AOF (créée en 1903, nommée William-Ponty à partir de 1915), on observe que l'ethnographie domine très largement chez les auteurs issus des premières promotions, qui écrivaient en réponse aux sollicitations de l'administration, la littérature faisant son apparition plus tardivement, en particulier sous l'influence de Charles Béart et de sa politique de promotion du théâtre (voir Traoré ; Mouralis). Cette perception de l'histoire en termes générationnels est d'ailleurs d'abord le fait des acteurs eux-mêmes : les textes autobiographiques de Sissoko, qui fourmillent de références à ses anciens camarades devenus cadres locaux, les identifient toujours par leur année de sortie de l'École normale; ils témoignent de ce sentiment d'appartenance à une « petite élite » d'anciens élèves organisée en promotions, appartenance aussi à une génération pionnière sur la voie de la modernisation - marque, s'il en était, de l'efficacité symbolique de cet aspect de la politique scolaire coloniale ${ }^{20}$. Moussa Travélé est ainsi invariablement présenté comme le "premier » : "le premier indigène qui ait rédigé une étude et un dictionnaire de sa propre langue ", "le premier à nous avoir donné un dictionnaire d'un format commode ", "le premier aussi à nous avoir dotés d'un dictionnaire français-bambara en même temps que bambara-français ", insiste Maurice Delafosse dans sa préface au Petit Dictionnaire français-bambara et bambara-français (Delafosse, Préface iv).

Pour les autorités coloniales, la trajectoire de Senghor (lui aussi «premier » agrégé de l'université d'origine africaine) s'inscrit donc dans le prolongement de celle de ses aînés. Élève brillant, originaire d'une famille aristocratique dont les contacts avec l'autorité coloniale française sont fréquents et anciens, plus tard naturalisé français (tout traits qu'il partage avec Hazoumé), il vient poursuivre ses études à Paris. Mais c'est dès lors un nouveau chapitre qui s'ouvre, dont l'action va se jouer sur deux fronts simultanément puisque, en même temps que se constitue progressivement en Afrique un espace public

\footnotetext{
à Paris dans le quartier de la Porte dorée, à la lisière du Bois de Vincennes. C'est une allusion au Musée des colonies, érigé en 1931 à l'occasion de l'Exposition coloniale internationale. Ce musée ethnographique, renommé « Musée de la France d'Outremer » en 1935, devient ainsi le symbole d'une mémoire culturelle reconstituée en métropole et redécouverte grâce à l'œuvre savante de la puissance impériale (Senghor, «Porte dorée » 10).

20. Voir par exemple, Sissoko 24, 25, 33, 43. Sur ce sentiment et l'homogénéité problématique de ce groupe, et sur la catégorie d'élite lettrée en général, voir Jézéquel, «Les enseignants».
} 
colonial où les voix indigènes sont autorisées et dont Senghor est partie prenante, sa réflexion rencontre celle des cercles intellectuels de la diaspora noire installée à Paris dans l'entre-deux-guerres. Cette histoire est mieux connue, mais on peut relever très brièvement, pour conclure, en quoi elle modifie le rapport à l'ethnologie de ces écrivains en devenir.

Tout d'abord, celle-ci apparaît comme une connaissance digne. C'est une discipline universitaire, avec diplômes et certificats, à l'égal de la sociologie ou de la philosophie, et non plus seulement un savoir pratique indexé sur un projet de connaissance et d'éducation des populations sous tutelle. La médiation des administrateurs disparaît, mais du même coup disparaît également l'expertise quasi "innée » que l'instituteur local a de sa propre population. La différence entre savoir amateur et savoir professionnel s'accuse comme le montrent par exemple le silence ostensible de l'école de Griaule à l'égard de l'ethnographie coloniale et, plus encore, sa méfiance à l'endroit des « Noirs évolués " soupçonnés d'avoir perdu le contact avec leurs racines. " On ne peut pas être à la fois à l'école et au bois sacré », professe Griaule dans ses cours à la Sorbonne à partir de 1943 (Griaule, Méthode 57), et c'est d'abord à ce primitivisme larvé que Césaire s'attaque dans sa diatribe contre les « ethnographes métaphysiciens et dogonneux » (voir de l'Estoile, «Au nom des vrais Africains "; Debaene, L'Adieu 428-30).

Pour l'intellectuel africain, le déplacement du monde de l'administration coloniale vers les réseaux plus politisés et plus internationalistes des Noirs de la capitale a également pour conséquence d'inscrire l'ethnologie dans une perspective plus internationale. C'est à Paris que Senghor est "saisi » par Frobenius (déjà traduit dans La Revue du monde noir) grâce à Césaire qui lui laissera son exemplaire de L'Histoire de la civilisation africaine (Senghor, " La révolution " $)^{21}$; c'est à Paris aussi qu'il envisage une thèse de linguistique sur les langues négro-africaines, après avoir suivi les cours de Lilias Homburger à l'EPHE ; c'est à Paris enfin qu'il découvre - sans doute après la guerre l'anthropologie de la personnalité américaine dont il se réclamera plus tard ${ }^{22}$.

Enfin, comme on l'a vu, l'arrivée à Paris est l'occasion d'une nouvelle inscription et d'une redéfinition de soi en termes raciaux, qui engendre une série de déplacements complexes, à la fois subjectifs et théoriques, dont Brent Edwards a retracé le cheminement, les conséquences et les contradictions. Notons simplement la rencontre d'une réflexion afro-américaniste qui,

21. Sur Frobenius et la négritude, voir Miller 16-21.

22. "La Négritude, c'est ce que les anglophones désignent sous l'expression de "personnalité africaine". Il n'est que de s'entendre sur les mots. » (Senghor, "Introduction" 8) Il est vraisemblable que cette découverte passe par le biais de l'ouvrage de Mellville Herskovits, Les Bases de l'anthropologie culturelle, traduit en français en 1952. 
dans le sillage de l'indigénisme des années 1920, connaît elle-même un " tournant ethnologique » après des élaborations davantage centrées sur la question de l'égalité des races ${ }^{23}$. Dans sa forme parisienne, cette réflexion s'enrichit des écrits ethnologiques d'administrateurs coloniaux antillais en poste en Afrique, qui contribuent à un repositionnement symbolique du continent, dont l'archaïsme supposé apparaît à la fois comme un retard et comme un signe d'authenticité qui le constitue en mémoire et en source. En réponse aux objections de Delafosse qui avait accusé Batouala d'insuffisance ethnographique, René Maran publie une longue étude consacrée à " certaines des croyances et coutumes en honneur chez les Bandas de l'Oubangui [où se passe l'action de Batouala] et les Saras du Tchad ». Au seuil de ce travail " qui se fonde sur treize années de recherche personnelles », il dénonce la plupart des écrits antérieurs sur l'Afrique équatoriale (ceux de Delafosse y compris) comme " trop souvent européanomorphiques ", accusant les auteurs d'avoir été incapables "de se faire une âme primitive " et " de se créer une mentalité indigène ». Il leur oppose l'exactitude et la technicité des études de Félix Eboué, Guyanais comme lui, «d'autant plus savoureuses » et dotées "d'autant plus de portée » que leur auteur « compte parmi les personnalités les plus représentatives de ces Noirs cent pour cent », à la fois haut fonctionnaire colonial « à l'entière satisfaction du ministère des Colonies » et ethnographe hors pair (Maran 326, 328).

$$
* * *
$$

Il est pertinent de s'interroger sur le « rapport à la littérature » des ethnologues métropolitains pour lesquels la littérature constitue une forme symbolique identifiée, qui peut jouer comme modèle ou contre-modèle (et souvent les deux à fois), mais la notion même de rapport à l'ethnologie, sur laquelle cet article est en partie fondé, est plus problématique. Elle suppose que le sujet qui s'exprime choisisse par dilection entre différents genres ou différentes formes de discours, et retienne l'une contre l'autre, préférant, par exemple, la fiction ou l'essai à la violence épistémique d'une « science impériale ». Il est dès lors tentant de rechercher dans ce que nous classons aujourd'hui comme de la littérature les signes d'une résistance ou, au contraire, d'une collaboration avec l'appareil colonial. Mais cela suppose des découpages, une organisation des discours et des possibilités de parole sans rapport avec la réalité historique de la domination. Pour le sujet colonisé, l'ethnographie n'a pas d'abord constitué un corps de savoir, ce fut plutôt un prisme envahissant qui déterminait une série de rapports : à l'autorité coloniale, à la collectivité, à l'histoire, à

23. L'expression «tournant ethnologique » est de Celius. Sur les évolutions de l'afroaméricanisme dans la première moitié du vingtième siècle, voir Aubrée et Dianteill. 
soi-même. Il est donc erroné de la considérer comme un genre auquel l'auteur aurait une relation instrumentale et uniquement stratégique. Mais c'est aussi, paradoxalement, ce qui la rapproche de la littérature au sens moderne car, quoique très encadrées, les pratiques discursives qu'elle a suscitées furent aussi l'occasion d'inventions de soi ; celles-ci furent à la fois des réponses à une demande extérieure et des lieux de construction de la subjectivité (voir Barthélémy 832-36, 847-52). C'est le paradoxe des dynamiques de savoir-pouvoir que d'ouvrir des possibilités par l'exercice de la contrainte.

L'abandon d'une poésie scolaire de "faiseur de vers » au profit d'une poésie lyrique et spiritualiste née des "retrouvailles avec les chants gymniques de sa culture serer » fut, comme on le sait, le parcours de Senghor (Diagne, Senghor 32). Son habileté a été d'en proposer, parallèlement, un récit particulièrement puissant, celui de la négritude, qui intègre les contributions ethnographiques indigènes comme une préhistoire : cette " littérature d'instituteurs » apparaissait comme la condition de possibilité d'une " renaissance » (Senghor, "Afrique noire » 233 ; voir Debaene, «La Littérature indigène »). L'efficacité de ce récit « civilisationnel » tenait à ce qu'il était appropriable à la fois par des penseurs révolutionnaires et par des auteurs de la diaspora noire, à la fois par Sartre et par Damas, qui allait reprendre en 1947, chez un éditeur d'avant-garde, les traductions des poésies rongué ou bassouto autrefois collectées par Delafosse mais débarrassées de tout appareil critique et redisposées typographiquement, artistement illustrées de gravures rupestres empruntées aux travaux de Frobenius (Damas, Poèmes nègres).

Mais la configuration discursive et politique dans laquelle s'inscrit historiquement l'itinéraire, à la fois subjectif et poétique, de Senghor allait produire d'autres trajectoires et d'autres œuvres, d'une variété, d'une complexité et d'une dispersion qu'il est difficile de ressaisir dans un récit unique. Comment lire les 510 pages de Doguicimi, écrites par le directeur de l'École régionale de Cotonou, descendant d'une famille de dignitaires de la cour du roi Sodji, lui-même opposant au royaume du Dahomey avant sa conquête par les Français ? Quel était pour Hazoumé le sens de son entreprise et comment celle-ci l'a-t-elle affecté en retour ? Il est certain en tout cas qu'une lecture en termes de collaboration ou de dissidence est insuffisante pour comprendre ce long roman historique qui est à la fois un hommage à la France, une défense de la civilisation dahoméenne, une leçon de morale chrétienne, une historicisation des sacrifices humains, et un plaidoyer pour l'ethnologie comme médiation entre sociétés et cultures (voir Riesz, Coundouriotis).

Comment lire les poésies bilingues que Jean-Joseph Rabearivelo écrivait au moment de son suicide, consécutif, selon plusieurs témoignages, à une énième fin de non-recevoir des autorités coloniales, qui avaient refusé de l'envoyer comme représentant de Madagascar à l'Exposition internationale de Paris de 1937 ? Comment lire les transformations du récit autobiographique 
de Mariama Bâ, entre la brève composition française parue en 1947 et le livre publié sous le même titre trente-deux ans plus tard (voir Barthélémy) ? Comment comprendre la hauteur aristocratique et l'espèce de véhémence qui émanent des écrits de Fily Dabo Sissoko, seules constantes d'une œuvre d'une grande variété générique, dans laquelle celui-ci se place constamment en position de corriger les discours européens sur les Noirs tout en considérant que la colonisation se trahit elle-même ? Sans renoncer intégralement à la catégorie de littérature, il faudrait sans doute en retarder l'usage, renoncer à fonder l'enquête sur elle, et y revenir au terme d'une histoire différenciée des modes de subjectivation par l'écriture.

Columbia University

\section{Euvres citées}

Archives de l'Institut d'ethnologie à la Bibliothèque centrale du Muséum d'histoire naturelle de Paris. AMH/IE/2AM 2.

Aubrée, Marion and Erwan Dianteill. " Misères et splendeurs de l'afroaméricanisme. Une introduction. "Archives de sciences sociales des religions 117 (janvier - mars 2002). Web September 20, 2013. < http:// assr.revues.org/2472,

Barthélémy, Pascale. "'Je suis une Africaine ... j’ai vingt ans' : écrits féminins et modernité en Afrique occidentale française (c. 1940-c. 1950) ». Annales. Histoire, sciences sociales, 4 (2009) : 825-52.

Barthes, Roland. "Bichon chez les Nègres. » Mythologies. Paris : Seuil, 1957.

Caillois, Roger. "Illusions à rebours. " La Nouvelle Nouvelle Revue française 24 (Décembre 1954): 1010-24, 25 (Janvier 1955): 58-70.

Célius, Carlo Avierl. "Cheminement anthropologique en Haïti. » Gradhiva 1 (2005). Web September 17, 2013. ‘http://gradhiva.revues.org/263 >

Césaire, Aimé. Discours sur le colonialisme. Paris: Présence africaine, 1955.

Coundouriotis, Eleni. Claiming History. Colonialism, Ethnography, and the Novel. New York: Columbia UP, 1998.

Comhaire-Sylvain, Suzanne. Les Contes haïtiens, origine immédiate et extension en Amérique. Port-au-Prince: Caravelle, 1937.

- Le Créole haïtien : morphologie et syntaxe. Port-au-Prince: Caravelle, 1936.

Congrès international et intercolonial de la Société indigène, 5-10 octobre 1931. Tome 2. Paris: École coloniale de Paris, 1931.

Conklin, Alice. A Mission to Civilize: The Republican Idea of Empire in France and West Africa, 1895-1930. Stanford: Stanford UP, 1997.

- In the Museum of Man: Race, Anthropology, and Empire in France, 1850-1950. Ithaca: Cornell UP, 2013. 
Damas, Léon-Gontran. Poèmes nègres sur des airs africains. Paris : GLM, 1947.

- Retour de Guyane. Éd. Sandrine Poujols. Paris: Jean-Michel Place, 2003.

Debaene, Vincent. L'Adieu au voyage. L'Ethnologie française entre science et littérature. Paris : Gallimard, 2010.

—. "Entre informateur et auteur. Discours ethnographique indigène et littérature en AOF. »Ethnologues en situation coloniale. Éd. Daniel Fabre, André Mary et Christine Laurière. Paris: CNRS Editions, à paraître (2016).

_ _ _ La 'Littérature indigène d'expression française': une histoire prépostcoloniale ». Éd. Vincent Debaene, Jean-Louis Jeannelle, Marielle Macé and Michel Murat. Paris : PUPS, 2013. 279-304.

Delafosse, Maurice. Broussard ou Les États d'âme d'un colonial suivis de ses propos et opinions. Paris : L'Harmattan, 2012.

—. Préface. Petit Dictionnaire français-bambara et bambara-français. Par Moussa Travélé. Paris : Paul Geuthner, 1913. iii-xiii.

Delobsom, Dim. L'Empire du Mogho-Naba: coutumes des Mossi de la HauteVolta. Paris: Domat-Montchrestien, 1932.

Diagne, Souleymane Bachir. L'Encre des savants. Réflexions sur la philosophie en Afrique. Paris: Présence africaine, Dakar: CODESRIA, 2013.

—_ Léopold Sédar Senghor: l'art africain comme philosophie. Paris: Riveneuve Editions, 2007.

Edwards, Brent Hayes. The Practice of Diaspora. Literature, Translation, and the Rise of Black Internationalism. Cambridge: Harvard UP, 2003.

Foucault, Michel. "Qu'est-ce qu'un auteur ? » Dits et écrits, tome 1 : 19541975. Paris: Gallimard, 2001. 817-49.

- Surveiller et punir. Naissance de la prison. Paris : Gallimard, 1975.

Hardy, Georges. Préface. Doguicimi. Par Paul Hazoumé. Paris: Larose, 1938. 9-11.

Hazoumé, Paul. Doguicimi. Paris: Larose, 1938.

- Le Pacte de sang au Dahomey. Paris : Institut d'ethnologie, 1937.

Griaule, Marcel. Dieu d'eau. Entretiens avec Ogotemmêli. Paris : Les Éditions du Chêne, 1948.

—. Méthode de l'ethnographie. Paris : PUF, 1957.

Jézéquel, Jean-Hervé. «Collecting Customary Laws: Educated Africans, Ethnographic Writings and the Making of Colonial Justice in French West Africa. "Intermediaries, Interpreters, and Clerks: African Employees in the Making of Colonial Africa. Eds B.N. Lawrence, E.L. Osborn and R. Roberts. Madison : U of Wisconsin P, 2006. 139-58.

__ «Les Enseignants comme élite politique en AOF (1930-1945). » Cahiers d'études africaines 178 (2005) : 519-43. 
"L'Organisation des cadres de l'enseignement en Afrique occidentale française (1903-fin des années 1930). » Genèses, 69(4) (2007) : 4-25.

- "Voices of Their Own? African Participation in the Production of Colonial Knowledge in French West Africa. " Ordering Africa: Anthropology, European Imperialism and the Politics of Knowledge. Eds. Helen Tilley and Robert Gordon. Manchester : Manchester UP, 2007. 145-72.

Keck, Frédéric. Lévy-Bruhl. Entre philosophie et anthropologie. Contradiction et participation. Paris: CNRS Éditions, 2008.

Laurière, Christine. Paul Rivet, le savant et le politique. Paris: Publications scientifiques du Muséum national d'histoire naturelle, 2008.

Lebel, Roland. L'Afrique occidentale dans la littérature française (depuis 1870). Paris: Larose, 1925.

l'Estoile, Benoît de. "Au nom des “vrais Africains”. Les Élites scolarisées de l'Afrique coloniale face à l'anthropologie (1930-1950). » Terrain. Carnets du patrimoine ethnologique 28 (mars 1997) : 87-102.

_. "Science de l'homme et domination rationnelle : savoir ethnologique et politique indigène en Afrique coloniale française. " Revue de synthèse 3-4 (2000): 291-323.

Lévi-Strauss, Claude. "Diogène couché. » Les Temps modernes 110 (mars 1955): 1-34.

Lüsebrink, Hans-Jürgen. La Conquête de l'espace public colonial. Prises de parole et formes de participation d'écrivains et d'intellectuels africains dans la presse à l'époque coloniale (1900-1960). Francfort-sur-le-Main : IKO-Verlag für Internationale Kommunikation, 2003.

Magloire, Gérarde, and Kevin A. Yelvington. « Haiti and the Anthropological Imagination. ", Gradhiva, 1 (2005). Web September 17, 2013. <http:// gradhiva.revues.org/335>

Maran, René. "Légendes et coutumes de l'Oubangui-Chari. » Euvres libres 147 (septembre 1933) : 325-81.

Midiohouan, Guy Ossito. L’Idéologie dans la littérature négro-africaine d'expression française. Paris : L'Harmattan, 1986.

_. "Littérature africaine: une critique de la critique. " Peuples noirs, Peuples africains 18 (1980): 75-88.

Miller, Christopher. Theories of Africans: Francophone Literature and Anthropology in Africa. Chicago: U of Chicago P, 1990.

Monod-Herzen, Gabriel. «Le Soudan français. » Europe 19:74 (15 février 1929): 246-61.

Mouralis, Bernard. «William-Ponty Drama. » European-Language Writing in Sub-Saharan Africa (1986). Ed. Albert S. Gérard. Amsterdam \& Philadelphia : John Benjamins Publishing. 130-40. 
Nardal, Paulette. "Eveil de la conscience de race. " La Revue du monde noir. Paris : Jean-Michel Place, 1992 : 343-49.

Piriou, Anne. "Indigénisme, et changement social : le cas de la revue OutreMer (1929-1937) ». L'Africanisme en questions. Éd. Anne Piriou et Emmanuelle Sibeud. Paris : Centre d'études africaines/éditions de l'EHESS, 1997. 43-69.

Poujols, Sandrine. Préface. Retour de Guyane. Par Léon-Gontran Damas. Paris: Jean-Michel Place, 2003. 13-17.

Pujarniscle, Eugène. Philoxène ou de la littérature coloniale. Paris : FirminDidot, 1931.

Racine, Daniel. Léon-Gontran Damas, l’homme et l'œuvre. Paris: Présence africaine, 1983.

Randau, Robert. Préface. L'Empire du Mogho-Naba: coutumes des Mossi de la Haute-Volta. Par Dim Delobsom. Paris: Domat-Montchrestien, 1932. i-vii.

Riesz, János. «From Ethnography to the African Novel : The Example of Doguicimi (1938) by Paul Hazoumé (Dahomey). " Research in African Literatures Vol. 35, No. 4 (Winter, 2004) : 17-32.

Said, Edward. « Representing the Colonized: Anthropology's Interlocutors. » Critical Inquiry Vol. 15, No. 2 (Winter, 1989) : 205-25.

«Les Sciences sociologiques au Collège de France ", La Revue du monde noir, J-M Place 1992 : 191.

Senghor, Léopold Sédar. "Afrique noire ». Les Plus Beaux Écrits de l'Union française et du Maghreb. Eds Mohamed el Kholti et al. Paris : La Colombe, Éditions du Vieux Colombier, 1947. 221-60.

—. "Introduction. " Liberté I. Négritude et Humanisme. Paris : Seuil, 1964. 4-10.

—. «Porte dorée. » Chants d'ombre. CEuvre poétique. Paris : Seuil, 1997.10. -. «La révolution de 1889 et Leo Frobenius. » Ethiopiques, 1982. Web May 17,2012. ‘http://ethiopiques.refer.sn/article.php3?id_article=1466〉

Sibeud, Emmanuelle. Une science impériale pour l'Afrique? La Construction des savoirs africanistes en France, 1878-1930. Paris : Éditions de l'EHESS, 2002.

Sidibé, Mamby. «La Chasse dans ses rapports avec les croyances religieuses, les mœurs et les coutumes indigènes au Birgo (colonie du Soudan Français, cercle de Kita). » Bulletin de l'Enseignement de l'AOF 67 (1929) : 60-78.

Sissoko, Fily Dabo. La Savane rouge. Avignon : Les Presses universelles. 1962.

Steins, Martin. «Entre l'exotisme et la négritude: la littérature coloniale. » Afrique littéraire 58 (1981) : 71-82.

Stoczkowski, Wiktor. Anthropologies rédemptrices. Le Monde selon LéviStrauss. Paris : Hermann, 2008. 
Warner, Tobias. "Para-Literary Ethnography and Colonial Self-Writing: The Student Notebooks of the William Ponty School. » Research in African Literatures. Forthcoming.

Wilder, Gary. The French Imperial Nation-State: Negritude and Colonial Humanism between the Two World Wars. Chicago: U of Chicago P, 2005. 